\title{
Estudo de vida real do perfil epidemiológico e da adesão ao tratamento de pacientes com asma alérgica grave em uso de omalizumabe durante 12 meses
}

\author{
Real-life study of the epidemiological profile and adherence \\ to the treatment of patients with severe allergic asthma \\ using omalizumab for 12 months
}

\author{
Fátima Chibana Soares ${ }^{1}$, Rafaela Rodrigues Sampaio Nogueira ${ }^{2}$, Lissa Sugahara ${ }^{3}$, \\ Pamela Passarelli ${ }^{3}$, Renato Kauffmann ${ }^{4}$, Alcindo Cerci Neto ${ }^{5}$
}

\section{Resumo}

\begin{abstract}
Introdução: a asma é uma doença heterogênea, caracterizada por inflamação crônica das vias aéreas inferiores, associada a diferentes fenótipos. O omalizumabe é utilizado em adição ao tratamento quando não se obtém o controle adequado da asma. Este estudo mostra o perfil epidemiológico e a adesão ao tratamento dos pacientes acompanhados no Ambulatório de Especialidades do Hospital Universitário da Universidade Estadual de Londrina (AEHU-UEL) em uso de omalizumabe em um período de 12 meses. Método: realizado um estudo transversal retrospectivo através de dados secundários de prontuário avaliando pacientes com diagnóstico de asma alérgica grave em uso de omalizumabe nos 12 meses prévios ao recrutamento. Resultados: foram selecionados 40 pacientes que preencheram os critérios de inclusão. A média de idade foi de 51,4 anos, com predomínio de mulheres (70\%), brancos (48\%), não tabagistas (90\%), com sobrepeso ou obesidade (75\%) e diagnóstico de asma na infância (45\%). O tempo médio de tratamento foi de 8,1 anos (DP 0,8). Havia comorbidades em $85 \%$ dos pacientes, com predomínio de rinite (62,5\%) e DRGE (40\%). Houve exacerbação em 29 pacientes levando a 8 internações (27,5\%); 93\% dos exacerbadores apresentaram faltas. Conclusão: a amostra é comparável a outros estudos de vida real nos achados epidemiológicos (idade, sexo, fenótipo, tempo de diagnóstico, controle da doença e tabagismo). O elevado número de faltas, assim como frequência de DRGE e outras comorbidades e a baixa adesão à terapêutica, podem justificar o elevado número de exacerbações e maior dificuldade de controle.
\end{abstract}

Palavras-chave: Asma alérgica. Omalizumabe. Adesão ao tratamento.

\footnotetext{
${ }^{1}$ Mestrado em Medicina e Ciências da Saúde pela Universidade Estadual de Londrina (UEL), Londrina, Paraná, Brasil. Professora Assistente do Departamento de Clínica Médica do Centro de Ciências da Saúde da Universidade Estadual de Londrina, Londrina, Paraná, Brasil.

2 Especialização em Clínica Médica pelo Hospital do Coração de Londrina, Londrina, Paraná, Brasil. Médica Plantonista no Pronto-socorro do Hospital do Coração de Londrina, Londrina, Paraná, Brasil. E-mail: rafaelaa_sampaio@hotmail.com

${ }^{3}$ Graduandas em Medicina na Universidade Estadual de Londrina, Londrina, Paraná, Brasil.

${ }^{4}$ Especialização em Clínica Médica pela Universidade Estadual de Londrina, Londrina, Paraná, Brasil. Médico Residente de Pneumologia da Prefeitura Municipal de Cruzeiro do Oeste, Cruzeiro do Oeste, Paraná, Brasil.

${ }^{5}$ Doutorado em Medicina e Ciências da Saúde pela Universidade Estadual de Londrina, Londrina, Paraná, Brasil. Professor Associado A do Departamento de Clínica Médica do Centro de Ciências da Saúde da Universidade Estadual de Londrina, Londrina, Paraná, Brasil.
} 


\begin{abstract}
Introduction: asthma is a hetereogeneous disease, characterized by chronic inflammation of the lower airways associated with different phenotypes. Omalizumab is used in addition to treatment when adequate asthma control is not achieved. This study shows the epidemiological profile and the adherence to the treatment of patients followed at the Medical Clinic of the State University Hospital of Londrina (AEHU-UEL) using omalizumab in the last 12 months. Methods: severe allergic asthma patients using omalizumab in the last 12 months were evaluated by means of secondary medical records. Results: forty patients were included and had complete medical record. The average age was 51.4 years mostly women $(70 \%)$, white $(48 \%)$, non-smoker $(90 \%)$, overweight or obese $(75 \%)$ and childhood asthma diagnosis $(45 \%)$. The average treatment time was 8.1 years (SD0.8). There were co-morbidities in $85 \%$ of the patients, mainly rhinitis in $62.5 \%$ and GERD in $40 \%$. There were exacerbations in 29 patients, leading to 8 hospitalizations $(27.5 \%), 93 \%$ of exacerbators was missed at least one time. Strong association with rhinitis $(p=0.07)$, and no disease control $(p=0.22)$. Conclusion: the sample is comparable to other real-life studies in almost all epidemiological findings (age, sex, phenotype, time of diagnosis, disease control and smoking). The high number of absences and frequency of GERD and other comorbidities, and poor adhesion, may justify the high number of exacerbations and more difficulty to control the disease.
\end{abstract}

Keywords: Severe allergic asthma. Omalizumab. Adherence.

\section{Introdução}

A asma é uma doença heterogênea, geralmente caracterizada por inflamação crônica das vias aéreas, cujo objetivo principal do tratamento é o controle dos sintomas por meio do uso de medicamentos contínuos. Apesar dos avanços nas estratégias de tratamento e do atual arsenal terapêutico, $10 \%$ a $15 \%$ dos pacientes que possuem formas mais severas da doença permanecem sem o controle adequado, a despeito de doses otimizadas de medicamentos como corticoides inalatórios (CI), broncodilatadores (BD), antagonistas de leucotrienos (AL) e corticoides orais $(\mathrm{CO}) .^{(1)} \mathrm{O}$ controle inadequado da asma se deve, além da própria gravidade da doença, à falha terapêutica, dificuldade no acesso à medicação, prescrição de doses inadequadas de medicamentos, comorbidades não tratadas ou exposição contínua aos alérgenos. ${ }^{(2)}$ Também é importante ressaltar que a baixa aderência ao tratamento impacta diretamente o seu controle. ${ }^{(3)}$

O medicamento omalizumabe foi o primeiro imunobiológico anti-imunoglobulina $\mathrm{E}$ (anti-IgE) de uso clínico, e que consiste em um anticorpo monoclonal humanizado anti-IgE. Este fármaco é utilizado como terapia "add-on", associado a CI em doses moderadas e altas, BD e AL em paciente com diagnóstico de asma alérgica persistente grave, que não obtém o controle adequado da doença. ${ }^{(4)}$ Há vários estudos demonstrando que o seu uso, principalmente em pacientes não obesos e menores de 65 anos de idade, reduz sintomas, melhora o controle da asma, aumenta a tolerância a exposições ambientais, melhora a realização de atividades diárias e promove redução dos episódios de exacerbação. ${ }^{(5)}$

O Ambulatório de Especialidades do Hospital Universitário de Londrina (AEHU-UEL) foi um dos primeiros centros de referência no Brasil a utilizar o omalizumabe em casos de asma de difícil controle. Este fármaco está sendo disponibilizado desde 2012 e, atualmente, o AEHU-UEL é centro de referência com o maior número de pacientes em uso do medicamento. É importante ressaltar que todo o tratamento é público e custeado pelo Estado. O objetivo deste estudo foi obter um perfil epidemiológico e clínico dos pacientes que fazem acompanhamento neste serviço e que estão em uso de omalizumabe por pelo menos 12 meses, e verificar o nível de aderência ao tratamento e o seu impacto no controle da doença. 


\section{Material e Métodos}

Em Londrina, Norte do Paraná, Brasil, os pacientes asmáticos são atendidos nas unidades básicas de saúde (UBSs) por profissionais de saúde previamente capacitados para identificar casos graves $^{(6)}$ e referenciá-los ao AEHU de acordo com os critérios contidos no Global Initiative for Asthma (GINA) versão 2016.(2) No AEHU, os pacientes diagnosticados como de difícil controle e com altas doses de CI iniciam o tratamento com omalizumabe, cuja dose é calculada de acordo com o preconizado pela literatura médica. As aplicações são realizadas no próprio ambulatório, alguns pacientes realizam aplicações a cada 15 dias e outros a cada 30 dias.

O omalizumabe está indicado a partir dos seis anos de idade, para paciente com asma alérgica moderada ou severa, que esteja com a asma ainda sem controle adequado apesar de estar no Step 4 de tratamento. O diagnóstico de asma alérgica é feito pelo teste cutâneo (prick test) ou dosagem de IgE; neste trabalho optamos fazer diagnóstico através da dosagem de IgE, pois através desta e do peso do paciente é calculada a dose da medicação e quantas vezes no mês será administrada. O omalizumabe está indicado para pacientes que apresentem IgE 30-1500 UI/mL.

Durante o segundo semestre de 2016 (julho a dezembro), todos os pacientes que já se encontravam em tratamento com omalizumabe por no mínimo 12 meses foram selecionados para o estudo. O projeto foi submetido ao comitê de ética de pesquisa em seres humanos da Universidade Estadual de Londrina (UEL), como parte do projeto de pesquisa CAAE 3838714.8.2004.523 e também CAAE 85694418.8.0000.5231. A amostragem foi por conveniência, e o único critério de inclusão no estudo foi estar em uso de omalizumabe. Foram excluídos pacientes que não completaram o tempo mínimo de tratamento de 12 meses consecutivos até dezembro de 2016. Todos os dados foram obtidos dos prontuários médicos por dois estagiários capacitados, de acordo com um formulário padronizado criado pelos pesquisadores responsáveis. Os dados de presença ou ausência na data aprazada foram extraídos dos controles de enfermagem, prontuário médico e registros de admissão no Hospital. Os dados faltantes nas análises eram confirmados com os pacientes pelos pesquisadores em consultas de rotina, desde que dentro do período de recrutamento, até dezembro de 2016.

Foram obtidos dados demográficos, informações sobre exacerbações, internações hospitalares, uso de antibiótico, admissão em Unidade de Terapia Intensiva (UTI) e visitas ao pronto atendimento. As informações eram computadas se ocorridas nos 12 meses prévios à coleta de dados. O tempo de utilização de omalizumabe era anotado de acordo com a primeira aplicação registrada no prontuário médico.

Foram considerados como pacientes não aderentes ao tratamento aqueles que faltaram à aplicação por duas ou mais vezes, no caso de aplicações mensais (a cada 30 dias), ou por mais de três vezes, no caso de aplicações a cada 15 dias.

Foi considerada como exacerbação a piora aguda dos sintomas respiratórios, tais como: dispneia, tosse, sibilância, ou a piora da função pulmonar (VEF1), avaliada por espirometria de acordo com o GINA 2016.

Para a análise dos dados foi utilizado o programa Epi Info para Windows versão 7.2 e o banco de dados foi criado no programa Excel 9.0. Os dados foram analisados por meio da estatística descritiva e inferencial. Inicialmente, foi testada a normalidade dos dados por meio do teste de ShapiroWilk. Variáveis contínuas foram expressas como média e desvio padrão (para o caso de distribuição gaussiana) e como mediana e interquartis (se distribuição não gaussiana). As variáveis categóricas expressas como proporção. Estatística descritiva foi utilizada para a apresentação de todas as variáveis relevantes. Os dados apresentados em gráficos e tabelas. O teste $t$ de Student, ou equivalente não paramétrico (Mann-Whitney) quando com distribuição não gaussiana, foi usado para a comparação das variáveis contínuas. As variáveis categóricas foram comparadas usando o 
teste de qui-quadrado de Pearson, com correção de continuidade de Yates. Realizada análise bivariada para variáveis preditoras do desfecho exacerbação. O nível de significância a ser utilizado foi de 5\% e as análises realizadas utilizando-se o programa Epi Info 7 (CDC, USA).

\section{Resultados}

$\mathrm{Na}$ data de obtenção de dados havia 52 pacientes em acompanhamento no AEHU, sendo que 5 deles não havia completado 12 meses de tratamento. Dos 47 pacientes que preenchiam os critérios de inclusão, 7 foram excluídos do estudo porque foram transferidos de outro serviço e não apresentavam prontuário médico completo ou informações médicas adequadas, o que inviabilizou o levantamento destas informações pelos pesquisadores.

Foram então incluídos 40 pacientes com asma alérgica grave na etapa 5 do tratamento. ${ }^{(2)} \mathrm{A}$ média de idade foi de 51,4 anos, com predomínio de mulheres (70\%), brancas (48\%), não tabagistas $(90 \%)$, com sobrepeso ou obesidade (75\%) e diagnóstico de asma na infância (45\%). Trinta e quatro pacientes $(85 \%)$ já tinham o diagnóstico de asma há mais de dez anos e grande parte havia sido exposta em algum momento da vida a irritantes ou alérgenos inalatórios $(50 \%$ no trabalho e $60 \%$ no domicílio através de fumaça produzida por fogão a lenha). A maioria dos pacientes apresentava algum tipo de comorbidade associada, sendo $85 \%$ com componente alérgico (rinite alérgica em $62,5 \%$, dermatite atópica 35\%, urticária 27,5\%, conjuntivite alérgica $22,5 \%$ e polipose nasal $17,5 \%$ ) e $75 \%$ a outras doenças (hipertensão arterial sistêmica $42,5 \%$, doença do refluxo gastroesofágico (DRGE) 40\%, transtorno depressivo 25\%, diabetes mellitus 20\%). O tempo de tratamento com uso de omalizumabe variou de 16 meses a 54 meses com uma média de 37 meses de uso (DP 10 meses). Após os 12 últimos meses de tratamento, $40 \%$ estavam controlados, $17,5 \%$ parcialmente controlados e $42,5 \%$ não controlados. Tabela 1 .

Tabela 1 - Dados epidemiológicos e demográficos da amostra analisada $(n=40)$.

\begin{tabular}{|c|c|c|c|c|}
\hline & Média & DP & Frequência & $\%$ \\
\hline Idade (anos) & 51,41 & 2,29 & & \\
\hline \multicolumn{5}{|l|}{ Sexo } \\
\hline Feminino & & & 28 & $70 \%$ \\
\hline Masculino & & & 12 & $30 \%$ \\
\hline \multicolumn{5}{|l|}{ Raça } \\
\hline Branco & & & 24 & $60 \%$ \\
\hline Negro & & & 3 & $7,5 \%$ \\
\hline Pardo & & & 11 & $27,5 \%$ \\
\hline Amarelo & & & 2 & $5 \%$ \\
\hline \multicolumn{5}{|c|}{ Exposição ocupacional a alérgenos } \\
\hline Sim, atualmente & & & 4 & $10 \%$ \\
\hline Sim, previamente & & & 16 & $40 \%$ \\
\hline Não & & & 20 & $50 \%$ \\
\hline \multicolumn{5}{|l|}{ Tabagismo } \\
\hline Sim, atualmente & & & 0 & $0 \%$ \\
\hline
\end{tabular}


Continuação

Sim, previamente

4

36

$1,4 \quad 0,72$

Carga tabágica (anos)

Exposição ao fogão a lenha

Sim, atualmente

Sim, previamente

Não

Peso (kg)

IMC (\%)

Idade ao diagnóstico

Infância

Adolescência

Adulto

Meia-idade

Terceira idade

Tempo de doença

Menos que 5 anos

Entre 5 e 10 anos

Entre 10 e 20 anos

Mais que 20 anos

Tempo de uso de omalizumabe (em anos)

Frequência de uso de omalizumabe

$15 / 15$ dias

$30 / 30$ dias

\section{Comorbidades}

Sim

Não

\section{Comorbidades específicas da asma}

Sim

Não

Fonte: Autores

Quanto a marcadores de controle e exacerbação, encontramos no período observado de 12 meses anteriores à coleta dos dados, 29 pacientes $(72,5 \%)$ que apresentaram ao menos uma exacerbação, $65 \%$ fizeram uso de esteroides

2,82

29,77

1,09

$75 \%$
18

1

11

9

1

$45 \%$

(n)

$2,5 \%$

$27,5 \%$

$22,5 \%$

$2,5 \%$

$12,5 \%$

$5 \quad 12,5 \%$

$6 \quad 15 \%$

$28 \quad 70 \%$

$3,14 \quad 0,12$
$0 \%$
$90 \%$

24

$60 \%$

16

$40 \%$

$30-75 \%$

$10 \quad 25 \%$

$34 \quad 85 \%$

$6 \quad 15 \%$ 
Tabela 2 - Dados relativos ao controle da asma nos últimos 12 meses prévios à coleta de dados (n=40).

\begin{tabular}{|c|c|c|c|}
\hline & Média (DP) & Frequência & Porcentagem \\
\hline \multicolumn{4}{|l|}{ Exacerbações } \\
\hline Sim & & 29 & $72,5 \%$ \\
\hline Não & & 11 & $27,5 \%$ \\
\hline Exacerbações & $1,27(0,7)$ & & \\
\hline \multicolumn{4}{|c|}{ Uso de corticoide oral } \\
\hline Sim & & 26 & $65 \%$ \\
\hline Não & & 14 & $35 \%$ \\
\hline \multicolumn{4}{|l|}{ Internação } \\
\hline $\operatorname{Sim}$ & & 8 & $20 \%$ \\
\hline Não & & 32 & $80 \%$ \\
\hline \multicolumn{4}{|c|}{ Uso de antibiótico } \\
\hline Sim & & 25 & $62,5 \%$ \\
\hline Não & & 15 & $37,5 \%$ \\
\hline \multicolumn{4}{|c|}{ Internação em UTI } \\
\hline Sim & & 1 & $2,5 \%$ \\
\hline Não & & 39 & $97,5 \%$ \\
\hline \multicolumn{4}{|l|}{ Pronto-socorro } \\
\hline $\operatorname{Sim}$ & & 14 & $35 \%$ \\
\hline Não & & 26 & $65 \%$ \\
\hline \multicolumn{4}{|c|}{ Consulta com especialista } \\
\hline $\operatorname{Sim}$ & & 12 & $30 \%$ \\
\hline Não & & 28 & $70 \%$ \\
\hline
\end{tabular}

Fonte: Autores

Dez pacientes foram considerados como não aderentes ao tratamento no último ano (25\%), sendo 7 no grupo quinzenal e 3 no grupo mensal. Considerando o grupo de aplicação quinzenal e anual de acordo com a definição usada, não houve diferença estatística significativa entre estes grupos $(\mathrm{p}=0,56)$.

Os pacientes foram divididos em dois grupos quanto à presença de exacerbações nos últimos 12 meses. Não houve diferença entre os grupos de exacerbadores e não exacerbadores quanto à idade, sexo, tempo de tratamento, início do diagnóstico, controle da doença ou frequência do uso de omalizumabe (Tabela 3). Dos 29 pacientes que exacerbaram, 13 deles apresentaram até duas exacerbações, 9 de duas a cinco exacerbações e 7 acima de cinco exacerbações no último ano. O grupo de exacerbadores, apesar de apresentar tempo de tratamento com omalizumabe semelhante ao grupo de não exacerbadores (3,2 - 3,1 anos), permaneceram não controlados a despeito do tratamento.

As características mais presentes nos pacientes exacerbadores foi o maior absenteísmo, menor aderência ao tratamento $(\mathrm{p}=0,04)$, permaneciam não controlados $(\mathrm{p}=0,22)$, apresentavam quadros de rinite alérgica $(\mathrm{p}=0,07)$ e associação a comorbidades $(\mathrm{p}=0,04)$. 
Tabela 3 - Dados epidemiológicos separados de acordo com a exacerbação ou não do paciente, nos 12 meses que precederam a coleta de dados $(n=40)$.

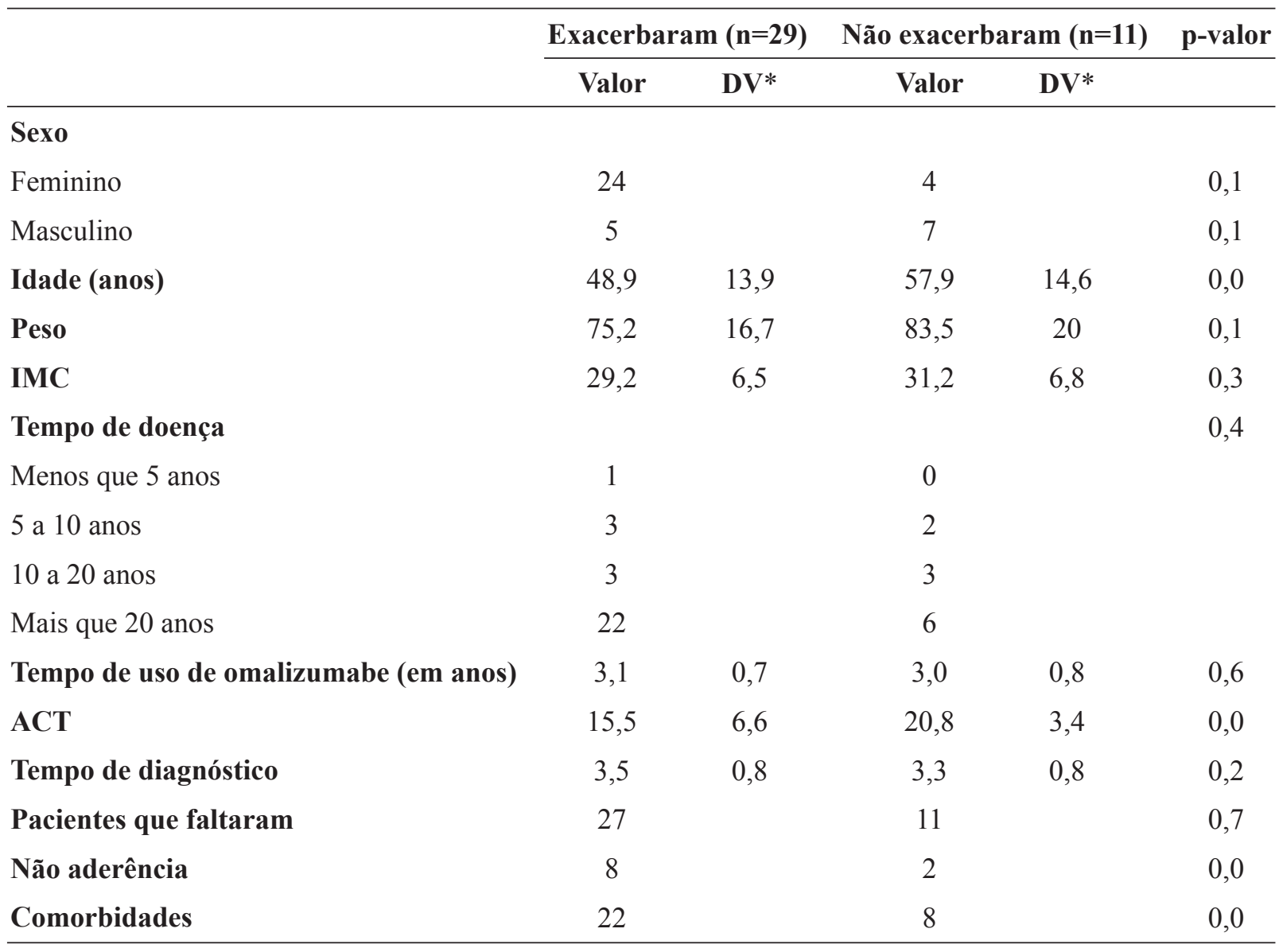

Fonte: Autores

*DV: desvio padrão.

\section{Discussão}

A capacitação realizada nas unidades básicas de saúde ${ }^{(3,6)}$ permite que exista um fluxo bem definido de diagnóstico e encaminhamento dos pacientes com asma de difícil controle. Entretanto, os pacientes passaram a fazer uso de omalizumabe em tempos diferentes: 1,4 anos a 4,5 anos. Contudo, neste ínterim houve informatização dos dados e houve grande perda de dados e informações destes pacientes.

A grande maioria da amostra foi composta por mulheres $(70 \%)$, fenótipos alérgicos $(85 \%)$, não controlados $(42,5 \%)$, não tabagistas $(90 \%)$ e com diagnóstico de asma na infância (45\%). A literatura documenta que pacientes com quadro clínico semelhante apresentaram as mesmas características. $^{(1,4,7)}$ Também foi demonstrado que estes pacientes mais graves têm elevado número de comorbidades alérgicas e não alérgicas associadas. $^{(1,4,7)}$ Em um estudo canadense que acompanhou pacientes de 14 a 55 anos de idade com diagnóstico de asma severa, os autores observaram que $77 \%$ dos pacientes apresentavam pelo menos uma comorbidade associada, ${ }^{(8)}$ à semelhança do presente estudo que encontrou $85 \%$ de pacientes com comorbidade alérgica e $75 \%$ com outras comorbidades clínicas. A doença predominante como comorbidade alérgica foi a rinite alérgica, e como comorbidade clínica a hipertensão arterial sistêmica.

Denlinger e colaboradores estudaram exacerbações em pacientes acima de seis anos, estabelecendo uma relação entre exacerbações 
frequentes e DRGE; ${ }^{(9)}$ assim, umas das possíveis justificativas para maior dificuldade no controle da asma e no maior número de exacerbações é a alta prevalência de DRGE nestes pacientes. Em nosso estudo, tivemos $40 \%$ dos pacientes com diagnóstico de DRGE.

Dos 40 pacientes estudados, 29 apresentaram ao menos uma exacerbação nos últimos 12 meses, com uma média de 4,5 exacerbações por paciente. Uma das possíveis causas está relacionada à observação de que em centros de referência em asma de difícil controle e com aplicação de imunobiológicos, os pacientes tendem a reduzir ou suspender o uso de CI e outros medicamentos. Outras questões relacionadas à adesão ao tratamento são as faltas e dias sem uso de omalizumabe.

Abraham e colaboradores publicaram uma revisão sistemática com 4.117 pacientes de 32 países, incluindo um centro brasileiro, sobre a efetividade de omalizumabe na vida real. ${ }^{(10)}$ Os estudos analisados forneceram evidências de que o tratamento com omalizumabe melhora significativamente parâmetros subjetivos e objetivos da função pulmonar, e também melhora significativamente o controle da asma em pacientes com asma alérgica grave. Houve redução significativa de exacerbações após 12 meses de tratamento, variando de $45,7 \%$ a $80 \%$; porém, estes pacientes persistiram apresentando de 2,6 a 3,2 exacerbações por ano. ${ }^{(5,10)}$ Assim, o número de exacerbadores persistentes após 12 meses de omalizumabe está acima do descrito pela literatura.

Existem outras justificativas para esta persistência do número de exacerbações no presente estudo. Na literatura, há relatos de estudos que encontraram um maior número de exacerbações em indivíduos acima dos 65 anos. ${ }^{(5)} \mathrm{E}$, entretanto, no presente estudo, observou-se que apenas nove pacientes tinham mais de 65 anos e quando analisada a idade e o número de exacerbações, obteve-se um resultado não significante estatisticamente, pelo contrário, com tendência a exacerbações nos mais jovens. Não houve diferença entre idade, gênero, tempo de tratamento e de diagnóstico com o risco de exacerbações. Apenas a presença de comorbidades se associou a uma maior chance de exacerbações, além da não adesão ao tratamento com omalizumabe.

O uso de imunobiológicos no Brasil progride lentamente, principalmente devido ao seu alto custo. Sabe-se que pacientes com asma grave consomem grande parte dos recursos públicos para o seu tratamento ${ }^{(11,12)}$ sendo necessários estudos de custo benefício e custo efetividade do uso de imunobiológicos. ${ }^{(3)}$ Aqui, o estudo demonstrou que mesmo com o fornecimento gratuito do omalizumabe, praticamente todos os pacientes apresentaram ao menos uma falta (38 pacientes ou 95\% da amostra) nos últimos 12 meses de tratamento.

Não há consenso na literatura sobre $o$ conceito de aderência ao tratamento, sendo que a maior parte dos estudos considera o paciente aderente aquele que utiliza ao menos $80 \%$ da dose do medicamento prescrito. ${ }^{(13)}$ Janson e colaboradores reportaram $83 \%$ de aderência em pacientes com aplicações quinzenais e $65 \%$ em pacientes com aplicações mensais, mas considerou como não aderentes os pacientes com perda de apenas uma aplicação. ${ }^{(14)}$

Este estudo não foi delineado para avaliar a efetividade do omalizumabe nos pacientes e não tem este poder estatístico.

A aderência ao tratamento está diretamente associada ao controle da doença e costuma ser baixa em pacientes asmáticos. ${ }^{(15)}$

\section{Conclusão}

O presente estudo avaliou pacientes com asma alérgica grave em uso de omalizumabe durante 12 meses, e foi possível concluir que o estudo teve o mesmo perfil epidemiológico de outros estudos de vida real com predomínio de mulheres, brancos, não tabagistas, com comorbidades. Além disso, os pacientes ainda apresentaram exacerbações, porém, quadros menos graves, em sua maioria sem necessidade de internação, e principalmente sem necessidade de permanência em Unidade de Terapia Intensiva. 
Dentre os pacientes que exacerbaram, houve maior número de faltas na aplicação do omalizumabe, menor aderência e maior número de comorbidades, o que demonstra a eficácia da medicação, a busca pelo melhor controle das comorbidades e a importância de orientar aos pacientes os benefícios em seguir rigorosamente a periodicidade das aplicações.

Apesar das limitações associadas ao delineamento do estudo, a amostra apresenta características semelhantes a outros estudos de vida real.

Foi possível observar também a necessidade de serem realizados mais estudos com modelo fármaco-econômico.

\section{Referências}

1 Barbosa MP, Almeida AB, Pereira C, Chen CW, Georgiou P, Peachey G. Real-life efficacy and safety of omalizumab in Portuguese patients with persistent uncontrolled asthma. Rev. Port. Pneumol. 2015;21(6):151-6.

2 Global Initiative for Asthma. Global strategy for asthma management and prevention. USA: GINA; 2016. p. 147.

3 Stelmach R, Cerci A Neto, Fonseca ACDCF, Ponte EV, Alves G, Araujo-Costa IN, et al. A workshop on asthma management programs and centers in Brazil: reviewing and explaining concepts. J. Bras. Pneumol. 2015;41(1).

4 Rubin AS, Souza-Machado A, Andrade-Lima M, Ferreira F, Honda A, Matozo TM. Effect of omalizumab as add-on therapy on asthmarelated quality of life in severe allergic asthma: a Brazilian study (QUALITX). J Asthma. 2012;49(3):288-93.

5 Sposato B, Scalese M, Latorre M, Scichilone $\mathrm{N}$, Matucci A, Milanese M, et al. Effects of omalizumab in severe asthmatics across ages: A real-life Italian experience. Respir Med. 2016;119:141-9.

6 Cerci A Neto. Mudança no perfil do manejo da asma em uma cidade brasileira de médio porte após programa estruturado: dado após quatro anos de implantação. [tese]. Londrina: Universidade Estadual de Londrina; 2009.
7 Dias JG, Silva P, Mendes A, Costa C, Pedro E, Barbosa MP. Omalizumabe no tratamento da asma brônquica alérgica: Experiência num serviço de imunoalergologia. Rev. Port. Imunoalergologia. 2012;20(4):263-71.

8 Chen W, Marra CA, Lynd LD, Fitzgerald JM, Zafari Z, Sadatsafavi M. The natural history of severe asthma and influences of early risk factors: A population-based cohort study. Thorax. 2016;71(3):267-75.

9 Denlinger LC, Phillips BR, Ramrtnam S, Ross $\mathrm{K}$, Bhakta NR, Cardet JC, et al. Inflammatory and comorbid features of patients with severe asthma and frequent exacerbations. Am J Respir Crit Care Med. 2017;195(3):302-13.

10 Abraham I, Alhossan A, Lee CS, Kutbi H, MacDonald K. "Real-life" effectiveness studies of omalizumab in adult patients with severe allergic asthma: Systematic review. Allergy Eur J Allergy Clin Immunol. 2016;71(5):593610.

11 Franco R, Campos H, Sarinho EC, Cerci A Neto. O custo da asma grave para o sistema público de saúde e para as famílias. Gaz Médica da Bahia. 2008;78(2):45-51.

12 Serra-Batlles J, Plaza V, Morejn E, Comella A, Brugues J. Costs of asthma according to the degree of severity. Eur Respir J. 1998;12(6):1322-6.

13 Osterberg L, Blaschke T. Adherence to medication. New Eng Journ Med. 2005; 353:487-97.

14 Janson SL, Solari PG, Trzaskoma B, Chen H, Haselkorn T, Zazzali JL. Omalizumab adherence in na observational study of patients with moderate to severe allergic asthma. Ann Allergy Asthma Immunol. [Internet]. 2015 June [cited 2018 Feb 4]; 114(6):516-21. Available in: http://www.ncbi.nlm.nih;gov/ pubmed/26021894

15 Wisnivesky JP, Lorenzo J, Lyn-Cook R, Newman T, Aponte A, Kiefer E, et al. Barriers to adherence to asthma management guidelines among inner-city primary care providers. Ann Allergy Asthma Immunol. 2008;101(3):26470. doi: 10.1016/S1081-1206(10)60491-7. 
Recebido em: 22 out. 2019 Aceito em: 1 jun. 2020 